\title{
M-resolutions and Deformations of Quotient Singularities
}

\author{
KURT BEHNKE ${ }^{1}$ \\ Jan ARThur Christophersen
}

INTRODUCTION

Due to the work in [Brieskorn], [Tjurina], [Artin], [Wahl 2] and [Lipman] one understands very well the notion of simultaneous resolution for rational surface singularities. Given a rational surface singularity $X$, there exists a smooth parameter space Res representing the functor of deformations of the (minimal) resolution. The family $\mathcal{Y} \rightarrow$ Res contracts to $\mathcal{X} \rightarrow$ Res. The fibers $Y_{t} \rightarrow X_{t}$ are the minimal resolutions of $X_{t}$. There is a finite and Galois map Res $\rightarrow \operatorname{Def}_{X}$ (the versal base space of $X$ ). The image is the Artin component,which represents the functor of deformations of $X$ with simultaneous resolution after finite base change, and the covering group $W$ is a reflection group which is also the monodromy group of the component.

In the case of rational double points (RDPs) $W$ is the Weyl group of the corresponding root system $\left(A_{k}, D_{k}, E_{6}, E_{7}\right.$ or $\left.E_{8}\right)$. In general much information about the deformation, e.g. the discriminant and adjacencies, may be read off the geometry of this covering. The reflections in $W$ are related to certain divisors, called roots, on $Y$ and their liftings. The contraction map is induced by contracting RDP-configurations on $Y$.

In connection with his work on deformations of cyclic quotient singularities (CQS), the second author observed a natural Galois covering of each component in the reduced versal base space of a CQS. In the case of the Artin component the observed covering group was the same as for Res $\rightarrow$ Def. (See [Christophersen 1] and [Christophersen 2].) The first author conjectured that this was the monodromy cover and we asked ourselves if there was a deformation theoretic explanation. The purpose of this paper is to answer this question.

We will show that something similar to the Res $\rightarrow$ Def picture actually happens for every non-embedded component of the versal base space of a quotient singularity. (For us a quotient singularity is the singularity of $\mathbb{C}^{2} / G$ where $G \subset \mathrm{GL}(2, \mathbb{C})$ is a finite subgroup which we can assume to be without pseudo-reflections.) For quotient singularities, the construction of the Artin component is a special case of a procedure involving deformations of certain modifications which we call M-resolutions.

The application of threefold theory to deformations of rational singularities as found in [Kollár-Shepherd-Barron] was important both for discovering M-resolutions and for the proofs of their properties. In fact, our results are anticipated in Theorem 3.5(a) of that paper. Still - the definitions and statement of the main result may be made without reference to that work. The relevant results from [Kollá-Shepherd-Barron] are postponed to $\S 1$.

Consider a smoothing of a normal surface singularity $X$ with smooth generic fiber $F$. The Milnor number of this smoothing is $\mu=\operatorname{rk} H_{2}(F)$ and depends upon the component of the versal base space of $X$ on which the smoothing appears.

\footnotetext{
${ }^{1}$ supported by a "Heisenberg-Stipendium" Be 1078/1-2 of the DFG
} 
Definition. A quotient singularity is of type $T_{0}$ if it admits a smoothing with Milnor number $\mu=0$, i.e. a smoothing with no vanishing cycles.

These singularities are classified (see $\$ 1$ ). The simplest example is the cone over the rational normal curve of degree 4 . The curve is a hyperplane section of the Veronese surface in $\mathbf{P}^{5}$. We get a smoothing of the surface singularity by "sweeping out the cone". Thus the Milnor fiber is $\mathbf{P}^{2}$ minus a quadric, which has $b_{2}=0$.

If a local deformation of a proper modification $Y$ of a quotient surface singularity $X$ restricts to $(\mu=0)$-smoothings of the singularities of $Y$, then the second betti number of the fibers is constant, so there is no monodromy. Hence if the blowing down map to the deformation space of $X$ is Galois, with Galois group $G$, then $G$ is the monodromy group of the contracted family. This motivates why we restrict the singularities on $Y$ to type $T_{0}$. Further we impose a minimality condition, expressed in terms of the canonical divisor.

DEFINITION. An M-resolution of a quotient singularity is a modification $f: Y \rightarrow X$ such that

(1) $Y$ has only singularities of type $T_{0}$.

(2) $K_{Y} \cdot E \geq 0$ for all $f$ exceptional curves $E$. ( $K_{Y}$ is nef relative to $f$.)

Notice that the minimal resolution is an M-resolution.

Let $\left\{Y_{i}\right\}$ be the singularities on an M-resolution, $Y$, of $X$ and set $\operatorname{Def}_{Y_{i}}^{0}$ to be the $\mu=0$ deformations. (This will turn out to be a smooth, one-dimensional irreducible component of the versal base space.) Let $\operatorname{Def}_{Y}^{0}$ be the preimage of $\prod \operatorname{Def}_{Y_{i}}^{0}$ under the natural (smooth) map $\operatorname{Def}_{Y} \rightarrow \prod \operatorname{Def}_{Y_{i}}$. The family over $\operatorname{Def}_{Y}^{0}$ contracts to a deformation of $X$ with no monodromy and admits, if you will, simultaneous M-resolution. Our main result is

THEOREM. Let $S$ be an irreducible non-embedded component of the versal base space of a quotient singularity $X$ and let $S_{n}$ be its normalization. For every such $S$ there exists a unique $M$-resolution $Y$ such that

(1) The natural map $\operatorname{Def}_{Y}^{0} \rightarrow \operatorname{Def}_{X}$ has $S$ as image and is a finite Galois covering of $S_{n}$.

(2) The covering group $W$ is a reflection group and is the monodromy group of $S$.

(3) The reflections of $W$ may be explained in terms of certain divisors on $Y$ called roots. In particular the reflection hyperplanes are exactly the subspaces where the roots lift.

The result appears in a more precise form as the three theorems 3.1.4, 3.3.2 and 3.4. The one to one correspondence between $M$-resolutions and components follows from a theorem of Kollár and Shepherd-Barron that establishes a one to one correspondence between components and $P$-resolutions. In fact $S_{n}$ may be identified with the so called $q G$-deformation of the P-resolution (to be explained in $\S 1$ ). In $\S 3$ we show that each $\mathrm{P}$-resolution, $Z$, is dominated by a unique M-resolution $f: Y \rightarrow Z$ with the property that $K_{Y}=f^{*} K_{Z}$.

The proof of the theorem falls in two parts. The first part (§2) is to prove the result when $X$ is a type $T$ singularity - these are the singularities allowed on P-resolutions and the component is $\mathrm{qG}$. This is our analog to the work on simultaneous resolution of rational double points [Brieskorn] ,[Tjurina]. The general result (§3) can now be proven 
by reducing to this case. The idea is essentially the same as in the construction of the Artin component outlined in [Wahl 2], substituting M-resolution for minimal resolution, and P-resolution for RDP-resolution. A number of arguments have to be added or changed though.

In the RDP case Lipman's theorem says that the deformations of the RDP resolution map injectively into deformations of the singularity ([Lipman]). In our case this last part is taken care of by Kollár and Shepherd-Barron. They prove at least that the map from the qG deformation space of the P-resolution maps finitely and generically $1-1$ to the corresponding component. In particular the families over the open sets with nonsingular fibers coincide. In the cyclic quotient case [Stevens] shows that the map is actually an injection, but he has an example of a dihedral singularity where one component actually is singular.

For the cyclic quotients we say explicitly what the monodromy group is over each component $(\S 4)$. For all the other quotient singularities complete lists of monodromy groups will be obtained immediately from a complete classification of their P-resolutions.

There are some obvious questions arising from these results. Does a similar result exist for all rational singularities? At the moment we do not know. Simple examples tell us that it will be much more complicated to determine the monodromy group. In another direction; will M-resolutions help in determining adjacencies, i.e. the singularities of the nearby fibers? The answer here is certainly yes, but some technical difficulties have yet to be solved.

Notation. To describe orbit spaces of cyclic groups we write e.g. $\mathbb{C}^{3} / \mathbf{Z}_{r}(a, b, c)$ which means that a generator of $\mathbf{Z}_{r}$ acts on $\mathbb{C}^{3}$ with matrix equal to $\operatorname{diag}\left(\zeta^{a}, \zeta^{b}, \zeta^{c}\right)$ where $\zeta$ is a primitive $r$ 'th root of unity.

As we already have done, we misuse the functor notation Def ${ }_{X}$ to mean also the versal deformation space, that is - the space pro-representing the deformation functor. The same notation is used for both singularities and global objects like resolutions. It should be clear from the context which deformation theory is involved.

Acknowledgements. We are grateful to Jan Stevens for many discussions and for correcting some of our too naive ideas. Our collaboration in Oslo and Hamburg has been supported by both the Norwegian Research Council for Science and the Humanities and the Deutsche Forschungsgemeinschaft through its Schwerpunkt Komplexe Mannigfaltigkeiten.

\section{§1. P-RESOlUtions AND TYPE $T$ SingulaRities.}

1.1. To understand $M$-resolutions we must first look at the $P$-resolutions introduced in [Kollár-Shepherd-Barron]. We recall definitions and results from that paper. For further developments and conjectures about general rational surface singularities see [Kollár].

1.1.1. Consider a one parameter deformation of a quotient singularity $X$. The total space, $\mathcal{X}$, is a normal 3-fold. The canonical ring of this 3-fold is finitely generated. Thus one has a canonical model $\mathcal{Y}$ with $K_{\mathcal{Y}}$ Q-Cartier ([Kollár-Shepherd-Barron,3.5]).

A one parameter smoothing of a quotient singularity is called $\mathrm{qG}$ if the total space has Q-Cartier canonical divisor. The singularity of the total space must then be terminal $([i b i d, 3.6])$. A component of the versal base space of a quotient singularity is called $\mathrm{qG}$ 
if it contains a one parameter qG smoothing. Finally a quotient singularity is called a $\mathrm{qG}$ singularity if it has a $\mathrm{qG}$ component in its versal base space. It can be shown that a quotient singularity is qG iff $K_{\tilde{X}}^{2} \in \mathbf{Z}$ where $\tilde{X}$ is the minimal resolution.

The special fiber of the canonical model of a one parameter smoothing is thus a modification with only qG singularities. We are led to study the deformations of such surfaces.

1.1.2. Rational double points (RDPs) are $\mathrm{qG}$ since $K_{\tilde{X}}^{2}=0$.

Definition. A quotient singularity $X$ is of type $T$ if $K_{\tilde{X}}^{2} \in \mathbf{Z}$ and $X$ is not an $R D P$.

(In [Kollár-Shepherd-Barron] RDPs are included in the definition of type T.)

Definition. (Kollár-Shepherd-Barron) A P-resolution of a quotient singularity $X$ is a partial resolution $f: Z \rightarrow X$ such that $Z$ has only singularities of type $T$ or RDPs and $K_{Z} . E>0$ for all $f$ exceptional divisors $E$.

1.1.3. It turns out that the type $T$ singularities have a unique qG component and this component is smooth. These are used to form a one to one correspondence between Presolutions and the components of the reduced versal base space of a quotient singularity. More precisely;

Theorem. ([Kollár-Shepherd-Barron,3.9]) Let $f: Z \rightarrow X$ be a P-resolution and $Z_{i}$ the singularities on $Z$. Let Def $_{Z_{\mathbf{i}}}^{\prime}$ be the $q G$ component of the versal base space of $Z_{i}$ and Def $_{Z}^{\prime}$ the preimage of $\prod$ Def $_{Z_{i}}^{\prime}$ under the natural map $\operatorname{Def}_{Z} \rightarrow \prod \operatorname{Def}_{Z_{i}}$. Let $F:$ Def $_{Z} \rightarrow$ Def $_{X}$ be the contraction map induced by $f$. Then

(1) $F\left(\right.$ Def $\left._{Z}^{\prime}\right)$ is an irreducible component of Def $_{X}$.

(2) If $Z, \tilde{Z}$ are two P-resolutions of $X$, not isomorphic over $X$, and $F, \tilde{F}$ are the corresponding maps of deformation spaces, then $F\left(\operatorname{Def}_{Z}^{\prime}\right) \neq \tilde{F}\left(\mathbf{D e f}_{\tilde{Z}}^{\prime}\right)$.

(3) Every component of the reduced versal base space of $X$ arises in this way

The subspace Def ${ }_{Z}^{\prime}$ is smooth, but the corresponding component need not be. (See e.g. [Stevens,7.2] for an example of a dihedral quotient with a singular component.) The restriction of $F$ to Def $_{Z}^{\prime}$ is generically one to one ([Kollár-Shepherd-Barron,3.19]) so $F$ is the normalization.

1.2. We describe now the type $T$ and type $T_{0}$ singularities. First of all it follows from e.g. [Looijenga-Wahl,4.11.2] that a type $T_{0}$ singularity must be type $T$ and that the $\mu=0$ smoothing is $\mathrm{qG}$.

The type $T$ singularities may be characterized in several ways. They are precisely the cyclic quotient singularities of the form $\mathbb{C}^{2} / \mathbf{Z}_{r^{2} s}(1, r s d-1)$ where $r, s, d$ are positive integers; $r \geq 2, s \geq 1$ and $(r, d)=1$.([Kollar-Shepherd-Barron,3.10].) The index of a type $T$ singularity $X$ is $r$; i.e. $\omega_{X}^{[r]}$ is free, and the canonical cover is an $A_{r s-1}$ singularity.

If we let $A_{r s-1}$ be the germ of $\left(x y-z^{r s}=0\right) \subset \mathbb{C}^{3}$ then the action of the covering group $\mathbf{Z}_{r}$ extends to $\mathbb{C}^{3}$ as $\mathbf{Z}_{r}(1,-1, d)$. Thus $X$ is the hypersurface section $x y-z^{r s}=0$ of the terminal singularity $\mathbf{C}^{3} / \mathbf{Z}_{r}(1,-1, d)$. This yields the $\mathrm{qG}$ smoothing.

The Milnor number, $\mu$, of this smoothing is easily calculated by comparing the Euler characteristic of the Milnor fiber of $A_{r s-1}$ with the fiber in our smoothing. Thus $1+\mu=$ 
$((r s-1)+1) / r$ and $\mu=s-1$. In other words the type $T_{0}$ singularities are exactly the type $T$ singularities with $s=1$; i.e. of the form $\mathbf{C}^{2} / \mathbf{Z}_{r^{2}}(1, r d-1)$. We will sometimes refer to type $T_{\mu}$ singularities.

1.3. We will need some facts about cyclic quotient singularities and their deformations. Details may be found in [Riemenschneider], [Arndt], [Christophersen 1], [Stevens] and [Christophersen 2].

1.3.1. Let $G$ be a finite cyclic subgroup of $\operatorname{GL}(2, \mathbb{C})$ without pseudo-reflections. Such a $G$ is similar to one generated by $\operatorname{diag}\left(\zeta_{n}, \zeta_{n}^{q}\right)$. Here $n>q>0,(n, q)=1$ and $\zeta_{n}$ is a primitive $n$ 'th root of unity. The cyclic quotient singularity (CQS) is the singularity of $\mathbb{C}^{2} / \mathbf{Z}_{n}(1, q)$.

Let $m=n-q$ and consider the unique continued fraction expansion

$$
\frac{n}{m}=a_{1}-\frac{1}{a_{2}-\frac{1}{\cdots-\frac{1}{a_{e-2}}} .}
$$

Define $c_{0}=n, c_{1}=m, c_{i+1}=a_{i} c_{i}-c_{i-1}$ and $d_{0}=0, d_{1}=1, d_{i+1}=a_{i} d_{i}-d_{i-1}$ for $i=1, \ldots, e-2$. Then the invariant ring $\mathbb{C}[u, v]^{G}$ is minimally generated as an algebra by $z_{i}=u^{c_{i}} v^{d_{i}}$ for $i=0,1, \ldots, e-1$, ([Riemenschneider, Satz 2]). Dually consider

$$
\frac{n}{q}=b_{1}-\frac{1}{b_{2}-\frac{1}{\cdots-\frac{1}{b_{k}}}} .
$$

The dual graph of the CQS looks like

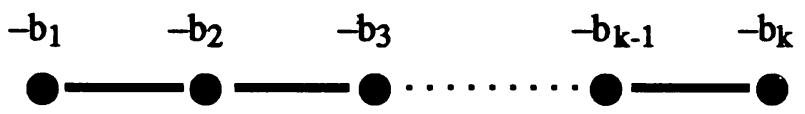

The torus action defines a $\mathbf{Z}^{2}$ grading on $\mathbf{C}[u, v]^{G}$, the homogeneous elements being the monomials in the $z_{i}$. Of course the weights of the generators are $w_{i}=\left(c_{i}, d_{i}\right)$. Thus a cyclic quotient singularity is determined by its multiplicities $a_{1}, \ldots, a_{e-2}$, and we will from now on use the notation $X\left(a_{1}, \ldots, a_{e-2}\right)$ for the singularity itself.

1.3.2. Let $k_{1}, \ldots, k_{n}$ be a sequence of $n$ non-negative integers. Such a sequence will be called a $n$-chain and denoted $\left[k_{1}, \ldots, k_{n}\right]$, often shortened to $[\mathbf{k}]$. Define now $n+2$ integers $\alpha_{i}=\alpha_{i}([\mathbf{k}])$ by $\alpha_{0}=0, \alpha_{1}=1$ and $\alpha_{i+1}=k_{i} \alpha_{i}-\alpha_{i-1}$ for $i=1, \ldots, n$. We say that [k] represents zero if $\alpha_{i}>0$ for $i=1, \ldots, n$ and if $\alpha_{n+1}=0$. This is equivalent to the continued fraction

$$
k_{1}-\frac{1}{k_{2}-\frac{1}{\cdots-\frac{1}{k_{n}}}}
$$


being both well-defined and actually equal to zero. The set of all such $n$-chains is denoted $K_{n}$. It is a finite set of cardinality $\frac{1}{n}\left(\begin{array}{c}2(n-1) \\ n-1\end{array}\right)$, the Catalan number.

If $X=X\left(a_{1}, \ldots, a_{e-2}\right)$, then define the set $K_{e-2}(X)=\left\{[\mathbf{k}] \in K_{e-2}: k_{i} \leq a_{i}\right.$ for all $i=$ $1, \ldots, r\}$. The main result on the versal deformation of cyclic quotients is the following. It is proven in [Stevens] where it appears as Theorem 4.1 and 5.1.

THEOREM. If $X\left(a_{1}, \ldots, a_{e-2}\right)$ is a cyclic quotient singularity then there is a one to one correspondence between $K_{e-2}(X)$ and the components of the reduced versal base, $S_{r e d}$, of $X$. Each irreducible component $S_{[\mathrm{k}]}$ is smooth.

The proof of this theorem in [Stevens] is based on the Kollár and Shepherd-Barron result quoted in 1.1.3. The possible $\mathrm{P}$-resolutions of $X$ are shown to be in one to one correspondence with $K_{e-2}(X)$.

1.3.3. Stevens observed ([Stevens,3.4]) that type $T$ singularities correspond to those chains representing zero $\left[k_{1}, \ldots, k_{e-2}\right]$ with only one $k_{i}$, say $k_{\delta}$, equal to 1 , by letting $a_{i}=k_{i}$ for $i \neq \delta$. Then $a_{\delta}=s+1$ in the above description. We may denote it $X([\mathbf{k}], s)$. In this setting the qG-component of $X([\mathbf{k}], s)$ corresponds to the chain $[\mathbf{k}]$.

To avoid introducing too much new notation, we will not refer to this description in the following. We would like to remark that one can find an explicit description of the qG family etc. in this set up using [Christophersen 2,2.1.1]. In $\S 2$ we will perform some computations for which this description is useful - this was how we have first found the results - but we leave it to the interested reader to work this out for herself.

1.4. We recall for future reference the intersection product of divisors on not necessarily smooth normal surfaces. Let $W$ be a normal surface, and $f: \tilde{W} \rightarrow W$ a resolution of singularities. For an effective (Weyl) divisor on $W$ let $D_{s}$ be its strict transform on $\tilde{W}$, and let $V$ be the unique $Q$-linear combination of $f$-exceptional curves such that $f^{*} D=D_{s}+V$ intersects each vertical curve trivially. Then the intersection product is

$$
\left(D^{\prime} \cdot D\right)_{W}=\left(f^{*} D^{\prime} \cdot f^{*} D\right)_{\tilde{W}}=\left(D_{s}^{\prime} \cdot\left(D_{s}+V\right)\right)_{\tilde{W}}
$$

[Giraud,2.4].

In the special case where $D_{s}$ is smooth, of self-intersection $-b$, and carries cyclic quotients $X_{i}=X\left(n_{i}, q_{i}\right)$ such that only their first exceptional component (in the order determined by the continued fraction expansion of $\left.n_{i} / q_{i}\right)$ intersects $D_{s},(D . D)_{W}=-b+\sum q_{i} / n_{i}$.

\section{§2. THE SPECIAL M-RESOLUTION FOR TYPE $T$ SINGUlARITIES.}

The purpose of this section is to construct a special M-resolution, $Y$, for a type $T$ singularity $X$, with the following properties. There is a smooth subspace $\operatorname{Def}_{Y}^{0}$ of $\operatorname{Def}_{Y}$ and a finite Galois map from $\operatorname{Def}_{Y}^{0}$ onto the qG-component of $\operatorname{Def}_{X}$. The covering group $W$ is a symmetric group. The family $\mathcal{Y}$ over $\operatorname{Def}_{Y}^{0}$ contracts to a deformation of $X$. We may think of this as simultaneous M-resolution after finite base change.

The reflection group $W$ is the monodromy group of the $\mathrm{qG}$-component, so $\operatorname{Def}_{Y}^{0}$ is the monodromy cover. Furthermore, on $Y$ we exhibit certain Weil divisors which we call "roots". For each reflection $\sigma \in W$, there exists a root $Z$ such that the reflection hyperplane of $\sigma$ is exactly the subspace where $Z$ lifts to the deformation of $Y$. 
2.1. Let $X=A_{r s-1} / \mathbf{Z}_{r}(1,-1, d)$ be of type $T$. Since $\mathbb{C}^{3} / \mathbf{Z}_{r}(1,-1, d)$ is an isolated 3 dimensional quotient singularity, it is rigid by [Schlessinger]. It follows from for example [Christophersen 3] that the deformations of $\mathrm{X}$ inside $\mathbb{C}^{3} / \mathbf{Z}_{r}(1,-1, d)$ form a smooth component in the versal base space of $X$. This is of course the qG-component, call it $S$. The tangent space of $S$ is $\left(T_{A_{r-1}}^{1}\right)^{\mathbf{Z}_{r}}$ (see [Kollár-Shepherd-Barron,3.18] or [Christophersen 3]).

A basis for $T_{A_{r,-1}}^{1}$ is $v_{0}, v_{1}, \ldots, v_{r s-2}$, where $v_{i}(f)=z^{i}$. The action of $\mathbf{Z}_{r}$ on $T_{A_{r s-1}}^{1}$ is defined by $v_{i} \mapsto \zeta_{r}^{-d i} v_{i}$, so the invariant subspace is spanned by $\left\{v_{c r}: c=0, \ldots, s-1\right\}$. Therefore $S \simeq\left(\mathbb{C}^{s}, 0\right)$ and the family over $S$ is defined by

$$
F=x y-z^{r s}-t_{s} z^{r(s-1)}-\cdots-t_{2} z^{r}-t_{1} \quad \text { in } \quad \mathbb{C}^{3} / \mathbf{Z}_{r}(1,-1, d) \times S .
$$

Here $t_{i+1}$ is dual to $v_{i r}$.

If we consider the last part of $F$ as a polynomial in $z^{r}$, we may write it as a product of its roots; i.e. set $G=x y-\prod_{i=1}^{s}\left(z^{r}-\xi_{i}\right)$. Thus $G$ defines a family $\mathcal{V}$ over the space $U \simeq\left(\mathbb{C}^{s}, 0\right)$ with coordinate functions $\xi_{i}$. The space $U$ maps to $S$ with covering group $W_{s}$, the symmetric group on $s$ letters. This is the covering we will study.

RemarK: The covering Res $\rightarrow$ Def of the $A_{r s-1}$ singularity looks similar to the above, but the connection is not immediate. Note that $U$ is not a subspace of Res.

2.2. We compute the action of $\mathbf{Z}_{r}$ on the Milnor homology $H_{2}(F)$ of $A_{r s-1}$. This yields the homology of the Milnor fiber of the type $T$ singularity $A_{r s-1} / \mathbf{Z}_{r}(1,-1, d)$ over the qG-component as $H_{2}(F)^{\mathbf{Z}_{r}}$, and it gives us the monodromy group.

2.2.1. By the Thom-Sebastiani property the Milnor lattices of the $A_{k}$ singularities in all positive dimensions are direct sums of the Milnor lattices of a zero-dimensional $A_{k}$ and a suitable $A_{1}$ singularity (sum of squares).([AGV, Theorem 2.9-2.11].) The group $\mathbb{Z}_{r}$ acts on both parts, and it is easy to see that it acts trivially on the class of the vanishing cycle of the $A_{1}$ singularity $\{x y=0\}$. In [AGV,Example 2.3] the reader can find an explicit computation of vanishing cycles for zero dimensional $A_{k}$. We have to change this only slightly to see how $\mathbf{Z}_{\boldsymbol{r}}$ acts.

The perturbation

$$
\tilde{f}=z^{r s}+\lambda z^{r} \quad \text { when } \quad|\lambda|<<1
$$

has $s$ critical values, which are $r$-fold degenerate. For $\tilde{f} \neq 0$ the critical values are even Morse. If we now choose $0<\epsilon<<|\lambda|$, comparison of the level sets

$$
\tilde{f}(z)=1 \quad \text { and } \quad \tilde{f}_{\epsilon}(z):=\tilde{f}(z)+\epsilon z=1
$$

( $\tilde{f}_{\epsilon}$ is a Morsification) shows that the $r s$ points of $\left\{z^{r s}=1\right\}$, the Milnor fiber of $A_{r s-1}$, can be grouped in $s$ subsets $\left\{z_{(i-1) r}, \ldots, z_{i r-1}\right\}(i=1, \ldots s)$ of $r$ points each such that

(1) $\mathbf{Z}_{r}$ rotates each group $\left(z_{(i-1) r} \mapsto z_{(i-1) r+1} \mapsto \cdots \mapsto z_{i r-1} \mapsto z_{(i-1) r}\right)$

(2) $\Delta_{1}=z_{0}-z_{1}, \Delta_{2}=z_{1}-z_{2}, \ldots, \Delta_{r s-1}=z_{r s-2}-z_{r s-1}$ is a distinguished basis of vanishing cycles. 
It is straightforward to write down the representation of $\mathbf{Z}_{r}$ on the $A_{r s-1}$ lattice. Let $A$ be the $(r-1) \times(r-1)$ matrix

$$
\left(\begin{array}{ccccc}
0 & 0 & \ldots & 0 & -1 \\
1 & 0 & \ldots & 0 & -1 \\
\vdots & \ddots & \ddots & & \vdots \\
0 & 0 & \ddots & 0 & -1 \\
0 & 0 & \ldots & 1 & -1
\end{array}\right),
$$

Then a suitable generator of $Z_{r}$ is represented by the following matrix:

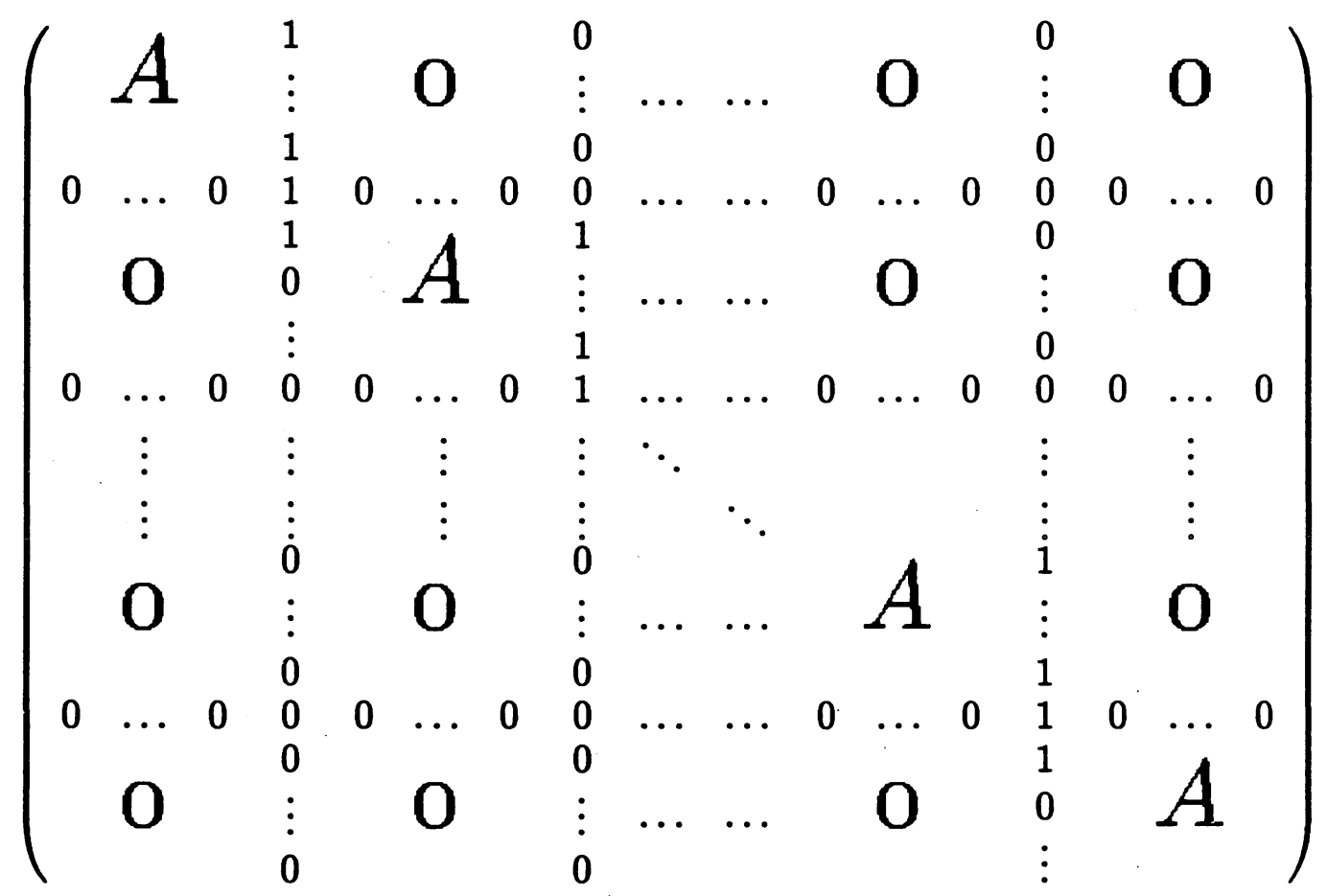

The character of the representation has value $r s-1$ at the unit element and -1 at all the other elements of $\mathbf{Z}_{r}$. In particular it decomposes as a sum of $s$ copies of each of the non-trivial irreducible representations and $s-1$ times the trivial one.

The $s-1$ generating invariant cycles are computed by summing $\frac{1}{r} \sum_{h \in \mathbf{Z}_{r}} h\left(\Delta_{i}\right)$. They are

$$
\Sigma_{i}=\Delta_{(i-1) r+1}+2 \Delta_{(i-1) r+2}+\cdots+r \Delta_{i r}+(r-1) \Delta_{i r+1}+\cdots+\Delta_{(i+1) r-1}
$$

for $i=1, \ldots, s-1$. The intersection numbers are

$$
\Sigma_{i}^{2}=-2 r \text { and } \Sigma_{i} \Sigma_{i+1}=r=\Sigma_{i} \Sigma_{i-1}
$$

and all other $\Sigma_{i} \Sigma_{j}=0$.

2.2.2. Hence the $\Sigma_{i}$ generate a root lattice of type $A_{s-1}$ which can be canonically identified with the second homology of the Milnor fiber of the type $T$ singularity. In passing to the quotient by $H$ all intersection numbers must be divided by $r$. So we have proven the first part of 
Proposition. On the $q G$-component of the versal base space of a type $T_{s-1}$ singularity the Milnor lattice is the root lattice of type $A_{s-1}$. The monodromy group is the full Weyl group of type $A_{s-1}$ acting in the usual way.

PROOF: To prove the latter assertion note that a monodromy transformation of the $A_{r s-1}$ lattice which fixes the $\mathbf{Z}_{\boldsymbol{r}}$ invariant sublattice, passes to a monodromy transformation in the quotient, and conversely. Thus the monodromy group of the $T_{s-1}$ singularity is the normalizer modulo the the centralizer of the invariant sublattice.

Embed the Weyl group representation of $A_{r s-1}$ into the permutation representation on $\mathbb{C}^{r s}$. In terms of the basis $z_{0}, \ldots, z_{r s-1}$ with $\Delta_{i}=z_{i-1}-z_{i}$ the generator of $Z_{r}$ is a product of $s$ cycles of length $r$ each. We may write

$$
\Sigma_{i}=z_{(i-1) r}+\cdots+z_{i r-1}-z_{i r}-\cdots-z_{(i+1) r-1}
$$

for $i=1, \ldots, s-1$. The subgroup of $S_{r s}$ which fixes each $\Sigma_{i}$ is exactly $S_{r} \times \cdots \times S_{r}$, each factor acting on a block $z_{(i-1) r}, \ldots, z_{i r-1}$ of variables. Modulo this subgroup the stabilizer of the invariant lattice is generated by the following elements (cycle notation)

$$
\sigma_{i}=\left(z_{(i-1) r}, z_{(i+1) r-1}\right)\left(z_{(i-1) r+1}, z_{(i+1) r-2}\right) \cdots\left(z_{i r-1}, z_{i r}\right)
$$

for $i=1, \ldots, s-1$. The transformation $\sigma_{i}$ maps $\Sigma_{i}$ to $-\Sigma_{i}, \Sigma_{i \pm 1}$ to $\Sigma_{i \pm 1}+\Sigma_{i}$ and fixes all the others. The group generated in this way is clearly the Weyl group of $A_{s-1}$.

2.3. Here we describe a special $M$-resolution of a type $T$ singularity.

2.3.1. Let $X=\mathbb{C}^{2} / \mathbf{Z}_{r^{2} s}(1, r s d-1)$ be of type $T$. The subgroup $\mathbf{Z}_{s}(1,-1)$ defines a $\mathbf{Z}_{r^{2}}$ covering $A_{s-1} \rightarrow X$. Let $M$ be the minimal resolution of the $A_{s-1}$ singularity.

PROPOSITION.

(1) $\mathbf{Z}_{r^{2}}$ acts on $M$ and the induced map on quotients $M / \mathbf{Z}_{r^{2}}=Y \rightarrow X$ is an $M-$ resolution.

(2) $Y$ has $s-1$ exceptional components $D_{i} \simeq \mathbf{P}^{1}(i=1, \ldots, s-1)$ and $s$ singular points $Y_{i}$ of type $\mathbb{C}^{2} / Z_{r^{2}}(1, r d-1)(i=1, \ldots, s)$. If we set $D_{0}$ and $D_{s}$ to be the preimages of the coordinate lines of the torus in $X$, then we may index so that $D_{i} \cap D_{i+1}=\left\{Y_{i+1}\right\}(i=1, \ldots, s-1)$ and $D_{i} \cap D_{j}=\emptyset$ if $j-i>2$.

(3) $K_{Y} \cdot D_{i}=0$ and $D_{i}^{2}=-\frac{2}{r^{2}}$. In particular if $C_{i, j}=\sum_{k=i}^{j-1} r D_{k}, i \leq j-1$, then $C_{i, j}^{2}=-2$.

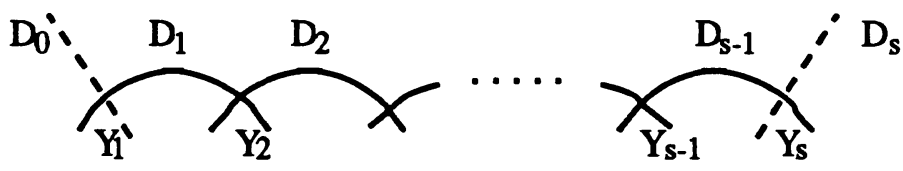

2.3.2. First we recall some well known facts about $M$, (denoted $M(2,2, \ldots, 2)(s-12$ 's) in [Laufer]). It is constructed by gluing $s$ copies of $\mathbb{C}^{2}, U_{1}, U_{2}, \ldots, U_{s}$, with coordinates $\left(x_{i}, y_{i}\right)$ and transition maps

$$
x_{i+1}=\frac{1}{y_{i}} \quad y_{i+1}=x_{i} y_{i}^{2} .
$$


The contraction map is defined on $U_{1}$ by

$$
x=x_{1} \quad z=x_{1} y_{1} \quad y=x_{1}^{s-1} y_{1}^{s}
$$

which extends over the whole of $M$.

Proof of 2.3.1: One checks that the action defined on $U_{1}$ by $x_{1} \mapsto \zeta_{r^{2}} x_{1}$ and $y_{1} \mapsto$ $\zeta_{r^{2}}^{r d-1} y_{1}$ not only extends to the other $U_{i}$ but is the same representation. It also makes the contraction map equivariant. This proves (2).

Since $\mathrm{Y}$ is the quotient of a manifold by a finite group we may compute intersections as in [Briney]. Also, as is well known $\omega_{Y}=\left(\omega_{M}\right)^{\mathbf{Z}_{r^{2}}}$. This yields (3). This shows that $Y$ is an M-resolution.

2.3.3. We realize now $Y$ as a hypersurface section of a 3 -fold with terminal quotient singularities. There is a $\mathbf{Z}_{r}$ action on $M \times \mathbb{C}$ :

$$
x_{i} \mapsto \zeta_{r} x_{i} \quad y_{i} \mapsto \zeta_{r}^{-1} y_{i} \quad z \mapsto \zeta_{r}^{d} z
$$

where $z$ is the coordinate for the line. The quotient has $s$ singularities, all of type $\mathbb{C}^{3} / \mathbf{Z}_{r}(1,-1, d)$ situated at the intersection of the exceptional $\mathbf{P}^{1} \times \mathbb{C}$.

Now $x_{i} y_{i}-z^{r}$ is a global invariant function on $M \times \mathbb{C}$ and therefore a function on the the quotient. The M-resolution $Y$ is the zero locus. Thus we have the following picture,

$$
\begin{array}{cccc}
Y & =\left\{x_{i} y_{i}-z^{r}=0\right\} \subset(M \times \mathbb{C}) / \mathbf{Z}_{r}(1,-1, d) \\
\downarrow & & \downarrow \\
X & =\left\{x y-z^{r s}=0\right\} \subset & \mathbb{C}^{3} / \mathbf{Z}_{r}(1,-1, d)
\end{array}
$$

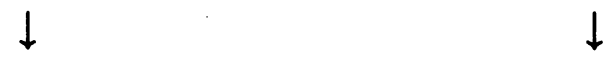

where the right vertical map is induced by

$$
x=x_{1} \quad y=x_{1}^{s-1} y_{1}^{s} \quad z=z .
$$

The total picture is seen in the following diagram

$$
\begin{aligned}
M & =M \\
\mathbf{Z}_{r} & \downarrow \\
B & \stackrel{\mathbf{Z}_{r}}{\longrightarrow} Y \subset(M \times \mathbb{C}) / \mathbf{Z}_{r}(1,-1, d) \\
\downarrow & \quad \downarrow \\
A & \stackrel{\mathbf{Z}_{r}}{\longrightarrow} X \subset \quad \mathbb{C}^{3} / \mathbf{Z}_{r}(1,-1, d)
\end{aligned}
$$

where $A$ (resp. $B$ ) is the canonical cover of $X$ (resp. $Y$ ).

2.4. Consider the the map $\operatorname{Def}_{Y} \rightarrow \prod \operatorname{Def}_{Y_{i}}$ and let $\operatorname{Def}_{Y}^{0}$ be the preimage of the product of the one dimensional $\mu=0$ (or qG) components of each $Y_{i}$. We shall compute the family over $\operatorname{Def}_{Y}^{0}=U$ and how it contracts to $\mathcal{V} \rightarrow U$. 


\subsubsection{We start with the}

Lemma. On the special $M$-resolution $Y$ of a type $T$ singularity $H^{1}\left(Y, \Theta_{Y}\right)=0$, in particular $\operatorname{Def}_{Y}^{0}=\prod \operatorname{Def}_{Y_{i}}^{0} \simeq\left(\mathbb{C}^{s}, 0\right)$.

Proof: It is well known that $\Theta_{Y}=\left(\Theta_{M}\right)^{\mathbf{Z}_{r^{2}}}$ so $H^{1}\left(Y, \Theta_{Y}\right)=H^{1}\left(M, \Theta_{M}\right)^{\mathbf{Z}_{r^{2}}}$. A simple computation shows that there are no invariants.

2.4.2. From the lemma we may identify $\operatorname{Def}_{Y}^{0}$ with $U$. Locally the $\mu=0$ deformations of the $Y_{i}$ are given by $x_{i} y_{i}-\left(z^{r}-\xi_{i}\right)=0$ in $\mathbb{C}^{3} / \mathbf{Z}_{r}(1,-1, d) \times U$. Let $\mathcal{Y}$ be the space covered by charts $\mathcal{Y}^{(i)}=\left\{x_{i} y_{i}-\left(z^{r}-\xi_{i}\right)=0\right\} \subset \mathbb{C}^{3} / \mathbf{Z}_{r}(1,-1, d) \times U, i=1, \ldots, s$. We may glue with

$$
x_{i+1}=\frac{x_{i}}{\left(z^{r}-\xi_{i}\right)} \quad y_{i+1}=y_{i}\left(z^{r}-\xi_{i+1}\right) \quad z=z
$$

on $\mathcal{Y}^{(i)} \cap \mathcal{Y}^{(i+1)}=\mathcal{Y}^{(i)} \cap\left\{y_{i} \neq 0\right\}$. Thus $\mathcal{Y}$ is a flat family over $U$ and is the total family over $\operatorname{Def}_{Y}^{0}$. The discriminant is $\prod \xi_{i}=0$-i.e. where at least one of the $Y_{i}$ is not smoothed out.

The family $\mathcal{Y} \rightarrow U$ contracts to the family $\mathcal{V} \rightarrow U$ defined in 2.1 .1 by setting

$$
x=x_{i} \prod_{j=1}^{i-1}\left(z^{r}-\xi_{j}\right) \quad y=y_{i} \prod_{j=i+1}^{s}\left(z^{r}-\xi_{j}\right) \quad z=z
$$

2.5. Consider the divisors $Z_{i, j}=\sum_{k=i}^{j-1} r E_{k}$ on $M$ and $C_{i, j}=\sum_{k=i}^{j-1} r D_{k}$ on $Y$. It is clear that $C_{i, j}=Z_{i, j} / \mathbf{Z}_{r^{2}}$. We call the $C_{i, j}$ the roots of the M-resolution $Y$.

2.5.1. We have already remarked that $C_{i, j}^{2}=-2$. Here are some more facts.

Proposition. Let $C=C_{i, j}$ be a root on the special $M$-resolution $Y=M / \mathbb{Z}_{r^{2}}$ of a type $T$ singularity and $Z$ the covering divisor on $M$. Let $\pi$ be the map from $M$ to $Y$. Then

(1) $h^{0}\left(\mathcal{O}_{C}\right)=1$ and $h^{1}\left(\mathcal{O}_{C}\right)=0$.

(2) $\mathcal{N}_{C / Y} \simeq\left(\pi_{*} \mathcal{N}_{Z / M}\right)^{\boldsymbol{Z}_{r^{2}}}$ where $\mathcal{N}_{C / Y}$ is the normal sheaf of $C$ in $Y$.

(3) There is an exact sequence

$$
0 \rightarrow \mathcal{N}_{C / Y} \rightarrow \mathcal{O}_{C} \rightarrow Q \rightarrow 0
$$

where $Q$ has support at the singular points of $Y_{i}$ and $Y_{j}$ with $Q\left(Y_{i}\right) \simeq Q\left(Y_{j}\right) \simeq \mathbb{C}$. (4) $h^{0}\left(\mathcal{N}_{C / Y}\right)=0$ and $h^{1}\left(\mathcal{N}_{C / Y}\right)=1$

Proof: (1) Assume first that $C$ is irreducible. Then $\mathcal{O}_{Z} \simeq \bigoplus_{k=0}^{r-1} \mathcal{O}_{\mathbb{P}^{1}}(k)$ as sheaf on $\mathbb{P}^{1}$. Computing invariants shows that $\mathcal{O}_{C} \simeq \mathcal{O}_{\mathbf{P}^{1}} \bigoplus(r-1) \mathcal{O}_{\mathbf{P}^{1}}(-1)$, so the statement is true for irreducible $C$.

Consider now $C=C_{i} \cup C_{i+1}$ and the exact sequence

$$
0 \rightarrow\left(I_{i}+I_{i+1}\right) / I_{i+1} \rightarrow \mathcal{O}_{C} \rightarrow \mathcal{O}_{C_{i}} \rightarrow 0
$$

where $I_{j}$ is the ideal of $C_{j}$ in $Y$. Since $I_{i}+I_{i+1}$ is the maximal ideal at the singular point of $Y_{i+1}$, the sequence reads

$$
0 \rightarrow \mathbf{m}_{\left(C_{i} \cap C_{i+1}\right)} \mathcal{O}_{C_{i+1}} \rightarrow \mathcal{O}_{C} \rightarrow \mathcal{O}_{C_{i}} \rightarrow 0
$$


and the result follows for this case also. We can obviously continue this way to prove the general statement.

(2) Since the group $G=\mathbf{Z}_{r^{2}}$ is finite we have $I_{C / Y}=\left(\pi_{*} I_{Z / M}\right)^{G}-$ thus $\mathcal{N}_{C / Y} \simeq$ $\mathcal{H}_{\mathcal{O}_{Y}}\left(\mathcal{I}_{C / Y}, \pi_{*} \mathcal{O}_{Z}\right)^{G}$. One can check that local restriction to $\left(\pi_{*} \mathcal{I}_{Z / M}\right)^{G}$ yields an injective (but not surjective) map $\pi_{*} \mathcal{N}_{Z / M} \rightarrow \mathcal{H o m}_{\mathcal{O}_{Y}}\left(\mathcal{I}_{C / Y}, \pi_{*} \mathcal{O}_{Z}\right)$. A local computation shows that the cokernel does not have invariants.

(3) The sequence is achieved by taking invariants of the exact sequence

$$
0 \rightarrow \mathcal{N}_{Z / M} \stackrel{e v}{\longrightarrow} \mathcal{O}_{Z} \rightarrow \operatorname{coker}(e v) \rightarrow 0
$$

where $e v$ is evaluation on the section $u_{k}^{r} v_{k}^{r}(k=i, \ldots, j)$ of $\mathcal{I}_{Z / M} / \mathcal{I}_{Z / M}^{2}$. In fact $e v$ is injective since where $Z$ is e.g. defined by $u^{r}, v$ is not a zero divisor in $\mathcal{O}_{Z}$ and vice versa. The statement about $Q$ follows now from a local computation.

(4) This follows from (1) and (3).

2.5.2. Let $R_{C} \subset U$ be the subspace of deformations of $Y$ to which a root $C$ lifts. (See the appendix for details). The roots and the reflections of $W$ are related by

Proposition. The subspaces $R_{C}$ where $C$ is a root of $Y$, are exactly the reflection hyperplanes of $W$.

Proof: By 2.5.1 and the appendix, $R_{C}$ is a hyperplane if there are no local obstructions for lifting $C$ and $\alpha: T_{0} U \rightarrow H^{1}\left(\mathcal{N}_{C / Y}\right)$ is not the zero map. The hyperplane $R_{C}$ is then $\operatorname{ker}(\alpha)$. The first statement is true in our case because the map $T_{Y}^{1}=H^{0}\left(\mathcal{T}_{Y}^{1}\right) \rightarrow H^{0}\left(\mathcal{T}_{C / Y}^{2}\right)$ factors through $H^{0}\left(\mathcal{T}_{Y}^{1}\left(\mathcal{O}_{C}\right)\right)$ and $T_{0} U \rightarrow H^{0}\left(\mathcal{T}_{Y}^{1}\left(\mathcal{O}_{C}\right)\right)$ is the zero map.

Let $C=C_{i, j}$. To compute $\alpha$ we first observe that $T_{0} U=H^{0}\left(\mathcal{T}_{B}^{1}\right)^{\mathbf{Z}_{r}}$, where $B$ is the canonical cover of $Y$. Secondly - using the same argument as in 2.5.1(2), one checks that $\mathcal{N}_{C / Y} \simeq\left(\mathcal{N}_{F / B}\right)^{\mathbf{Z}_{r}}$ (so $\left.H^{1}\left(\mathcal{N}_{C / Y}\right) \simeq H^{1}\left(\mathcal{N}_{F / B}\right)^{\mathbf{Z}_{r}}\right)$ where $F \subset B$ is the (Cartier) divisor covering $C$. I.e. $F$ is locally defined by the ideals

$$
\begin{aligned}
& \left(y_{i}^{r}\right) \text { in } \mathcal{O}_{B^{(i)}} \\
& \left(x_{k}^{r} y_{k}^{r}\right) \quad \text { in } \quad \mathcal{O}_{B^{(k)}}, k=i+1, \ldots, j-1 \\
& \left(x_{j}^{r}\right) \text { in } \mathcal{O}_{B^{(j)}}
\end{aligned}
$$

where $B^{(i)}$ are the charts of $B$ and $\mathcal{O}_{B^{(k)}}=\mathcal{O}_{U^{(k)}}^{Z_{r}}$. Thus $C$ is defined by the invariants of these ideals.

Let $\left(\xi_{1}, \ldots, \xi_{s}\right) \in T_{0} U \subset H^{0}\left(\mathcal{T}_{B}^{1}\right)$. If we just set $z$ to be the function $x_{i} y_{i}$, the corresponding lifting yields the following Cech cocycles of $\mathcal{N}_{C / Y} \simeq\left(\mathcal{N}_{F / B}\right)^{\boldsymbol{Z}_{r}}$ :

(1) on $F^{(i)} \cap F^{(k)}, k>i$ with coordinate ring $\mathrm{C}\left[x_{i}^{r}, x_{i}^{-r}, z\right] /\left(z^{r}\right) ; z^{r} \mapsto \xi_{i}$

(2) on $F^{(j)} \cap F^{(k)}, k<j$ with coordinate ring $\mathbb{C}\left[y_{i}^{r}, y_{i}^{-r}, z\right] /\left(z^{r}\right) ; z^{r} \mapsto \xi_{j}$.

In particular the kernel of $\alpha$ is exactly the set where $\xi_{i}=\xi_{j}$.

\section{§3. M-Resolutions and Monodromy in General}

3.1. We shall show in this section that for each $\mathrm{P}$-resolution of a quotient surface singularity there is a unique $\mathrm{M}$-resolution which dominates it, and such that all the vertical curves have trivial intersection with the canonical divisor. 
3.1.1. Observe that on a P-resolution we cannot blow up smooth points if we want to keep the canonical divisor numerically effective. So all we have to do is to show that a singularity of type $T$ has at most one M-resolution with the additional property that the canonical divisor intersects all exceptional curves trivially. We start with a Lemma that computes the necessary $\mathbf{Q}$-intersection numbers (cf. 1.4).

3.1.2. Let $X$ be a cyclic quotient singularity with exceptional components $E_{1}, \ldots, E_{k}$ on the minimal resolution $\pi: \tilde{X} \rightarrow X$. We use the notation of 1.3.1.

Consider the sequences of non-negative integers

$$
\begin{aligned}
& \alpha_{0}=n, \alpha_{1}=q, \quad \ldots \quad \alpha_{i+1}=b_{i} \alpha_{i}-\alpha_{i-1}, \quad \ldots \quad \alpha_{k}=1, \alpha_{k+1}=0 \\
& \beta_{0}=0, \beta_{1}=1, \quad \ldots \quad \beta_{i+1}=b_{i} \beta_{i}-\beta_{i-1}, \quad \ldots \quad \beta_{k}=q^{\prime}, \beta_{k+1}=n
\end{aligned}
$$

where $q^{\prime}$ is the unique number with $q q^{\prime} \equiv 1(n), 0<q^{\prime}<n$. Then one may check that (see e.g. [Knöller]) for $\kappa_{i}=\frac{\alpha_{i}+\beta_{i}}{n}-1, i=1, \ldots, k$, the canonical divisor $K_{\tilde{X}} \in \operatorname{Pic}(\tilde{X}) \otimes \mathbb{Q}$ is

$$
K_{\tilde{X}}=\sum_{i=1}^{k} \kappa_{i} E_{i} .
$$

In particular for a type $T$ singularity $\mathbb{C}^{2} / \mathbf{Z}_{r^{2} s}(1, d r s-1)$ note that $\kappa_{1}=\left(\frac{d}{r}-1\right), \kappa_{k}=$ $\left(\frac{r-d}{r}-1\right)$, and $\kappa_{1}+\kappa_{k}=-1$. This equality even characterizes the type $T$ singularities among all cyclic quotients.

3.1.3. Let now $Y$ be a normal modification of a quotient singularity $X$ such that $K_{Y}$ is numerically effective, and $Y$ has only singularities of type $T$. Let $E_{1}, \ldots, E_{t}$ be the exceptional components of $Y$. On the minimal resolution $\tilde{Y}$ of $Y$ denote by $\tilde{E}_{i}$ the strict transform of $E_{i}$.

LEMMA. $K_{Y} \cdot E_{i}=0$ if and only if $\tilde{E}_{i} \cdot \tilde{E}_{i}=-1$, and $E_{i}$ carries exactly two singular points of $Y$ with same $r, s, d$, such that on $\tilde{Y}$ the strict transform intersects their exceptional chains transversely in opposite ends.

Proof: Let for simplicity $E=E_{i}$, and assume $K_{Y} \cdot E=0$. Let $p_{1}, \ldots, p_{n}$ be the singular points on $E$, and let $A^{(i)}$ be the exceptional chain which resolves $\left(Y, p_{i}\right)$, with $A_{1}^{(i)}$ the irreducible component which intersects $\tilde{E}$. Let $K^{(i)}=\sum_{j} \kappa_{j}^{(i)} A_{j}^{(i)}$ be the canonical $\mathbb{Q}$ divisor for $\left(Y, p_{i}\right)$ on $A^{(i)}$.

Then

$$
\begin{aligned}
\left(K_{Y} \cdot E\right)_{Y} & =\left(K_{\tilde{Y}} \cdot \tilde{E}\right)_{\tilde{Y}}-\sum_{i=1}^{n}\left(K^{(i)} \cdot \tilde{E}\right)_{\tilde{Y}} \\
& =b-2-\sum_{i=1}^{n} \kappa_{1}^{(i)}
\end{aligned}
$$

Since $-1<\kappa_{1}^{(i)} \leq 0$ for all $i$ this intersection number can be zero only if $b=-\tilde{E} . \tilde{E}=1$. Then $\sum_{i=1}^{n} \kappa_{1}^{(i)}=-1$, and if $\left(Y, p_{i}\right)$ has invariants $n_{i}=r_{i}^{2} s_{i}, q_{i}=d_{i} r_{i} s_{i}-1$ this means $\sum_{i=1}^{n} \frac{r_{i}-\bar{d}_{i}}{r_{i}}=1$. 
By negative definiteness of the intersection matrix we have

We get

$$
\sum_{i=1}^{n}\left(\frac{d_{i}}{r_{i}}-\frac{1}{n_{i}}\right)=\sum_{i=1}^{n} \frac{q_{i}}{n_{i}}<1 .
$$

and hence

$$
\sum_{i=1}^{n} \frac{r_{i}-d_{i}}{r_{i}}+\sum_{i=1}^{n} \frac{1}{n_{i}}>n-1
$$

$$
\sum_{i=1}^{n} \frac{1}{n_{i}}>n-2
$$

The smallest possible value for $n_{i}$ for a type $T$ singularity being 4 this implies $n \leq 2$. On the other hand $n=1$ is clearly impossible. The converse is trivial.

3.1.4. Now it is easy to prove our uniqueness assertion.

THEOREM. For a singularity $X$ of type $T$ there exists a unique $M$-resolution $f: Y \rightarrow X$ such that the canonical divisor $\left(K_{Y} \cdot E\right)_{Y}=0$ for each exceptional curve $E$. In particular each $P$-resolution $Y$ of a quotient surface singularity has a unique $M$-resolution $f: Y \rightarrow Z$ with $f^{*} K_{Z}=K_{Y}$.

REMARK: We will call this M-resolution the unique crepant M-resolution.

Proof: The second assertion is an immediate consequence of the first. To prove that one, given an M-resolution $Y \rightarrow X$ of a type $T$ singularity with numerically trivial canonical divisor 3.1.2 tells us what $Y$ looks like. The exceptional curve is a chain of $s$ smooth rational curves with $s+1$ singularities $\mathbb{C}^{2} / \mathbf{Z}_{r^{2}}(1, d r-1)$ for some value of $r$ and $d$ as in 2.3.1. We know already that such a chain contracts to an $\mathbb{C}^{2} / \mathbf{Z}_{r^{2}}(1, d r s-1)$-singularity. So $r, s$ and $d$ are already fixed by $X$.

Let $\tilde{Y} \rightarrow Y$ be the minimal resolution of $Y$, and let $\tilde{Y}_{1} \rightarrow Y_{1}$ be the minimal resolution of a second M-resolution of the same $X$ with the same properties. Since the combinatorial structures of $Y$ and of $Y_{1}$ coincide, the dual graphs of $\tilde{Y}$ and $\tilde{Y}_{1}$ look the same. So both can be blown down to minimal resolutions of $X$ by contracting corresponding curves at each step. An isomorphism of the two resulting minimal resolutions can be traced back through the construction to yield an isomorphism between $\tilde{Y}$ and $\tilde{Y}_{1}$, and hence $Y$ and $Y_{1}$ are isomorphic.

3.2. Using the device 1.4 we compute the intersection numbers of exceptional curves on M-resolutions. Let $f: Y \rightarrow Z$ be the unique crepant $\mathrm{M}$-resolution of a $\mathrm{P}$-resolution as in 3.1.4. Assume that an $f$-exceptional curve $E$ maps to a singularity of type $T$ on $Z$. Then it has $E_{s}^{2}=-1$ on the minimal resolution $\tilde{Y}$ of $Y$, and carries exactly two singular points $\mathbb{C}^{2} / \mathbf{Z}_{r^{2}}(1, d r-1)$, and $\mathbb{C}^{2} / \mathbf{Z}_{r^{2}}(1,(r-d) r-1)$. Hence

$$
(E . E)_{Y}=-1+\frac{d r-1}{r^{2}}+\frac{(r-d) r-1}{r^{2}}=-2 / r^{2}
$$

and $(r E)^{2}=-2$. Obviously the only irreducible (-2)-cycles are these curves and possibly nodal curves which contract to rational double points on the M-resolution.

Similarly one computes for two adjacent such components $(r E)\left(r E^{\prime}\right)=1$, and for a chain $Z=r\left(E_{1}+\ldots E_{r}\right), Z^{2}=-2$. We leave it as an exercise to check that $\omega_{Y} . Z=0$. 
3.3. We now show that the local computations for the special M-resolution of type $T$ singularities, and of rational double points, patch together.

3.3.1. To this end we consider a P-resolution $f: Z \rightarrow X$ of a quotient surface singularity, and let $g: Y \rightarrow Z$ be the crepant M-resolution of $Z$. Let $z_{1}, \ldots, z_{t}$ be the singular points on $Z$, and for $i=1, \ldots, t$ let $E_{i}$ be the $g$-exceptional curve over $z_{i}$. We denote by $Z_{i}=\left(Z, z_{i}\right)$ the singularity of $Z$ at $z_{i}$, and by $Y_{i}=\left(Y, E_{i}\right) \cong g^{-1}\left(Z, z_{i}\right)$ the germ of $Y$ along the compact analytic set $E_{i}$. In both cases we use the same notation for a good representative of the germ.

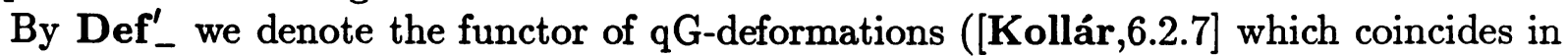
our case with the functor of $\omega^{*}$-constant deformations defined in [Wahl 1]. The latter functor is unobstructed for normal surfaces. We already know what the spaces are. The space $\operatorname{Def}_{Z_{i}}^{\prime}$ is the qG-component if $\left(Z, z_{i}\right)$ is of type $T$, and is the whole versal base space if $\left(Z, z_{i}\right)$ is an RDP. Thus Def ${ }_{Z}^{\prime}$ is the space defined in 1.1.3. The space $\operatorname{Def}_{Y}^{\prime}$ is the $\operatorname{Def}_{Y}^{0}$ described in the introduction. The spaces $\operatorname{Def}_{Y_{i}}^{\prime}$ are $\operatorname{Def}_{Y_{i}}^{0}$ if $\left(Z, z_{i}\right)$ is of type $T$ and $\operatorname{Res}$ if $\left(Z, z_{i}\right)$ is an RDP.

The contraction map $\varphi: \operatorname{Def}_{Z}^{\prime} \rightarrow \operatorname{Def}_{X}$ induced by $f$ is finite and generically one to one onto the irreducible component $S$ corresponding to $f$, as shown by [Kollár-ShepherdBarron]. If $D \subset S$ is the discriminant then $\operatorname{Def}_{Z}^{\prime} \mid \varphi^{-1}(D) \cong S \backslash D$, and their fundamental groups coincide. The restriction of the semiuniversal families $\mathcal{Z} \rightarrow \operatorname{Def}_{Z}^{\prime}$ and $\mathcal{X} \rightarrow$ Def $_{X}^{\prime}$ coincide too, and in particular have the same smooth fiber, and the same monodromy.

3.3.2. By 2.3.3 the contraction map from deformations of $Y$ to deformations of $Z$ maps $\operatorname{Def}_{\left(Y, E_{i}\right)}^{\prime}$ finite to one to $\operatorname{Def}_{\left(Z, z_{i}\right)}^{\prime}$. Since the qG property is local $\operatorname{Def}_{Y}^{\prime}$ maps finite to one to $\operatorname{Def}_{Z}^{\prime}$ too.

To each of the singularities $Z_{i}$ of the P-resolution $Z$ we associate its special monodromy group $W_{i}$. It is the monodromy group of the special component, as constructed in $\S 2$, if $Z_{i}$ is of type $T$ and is the classical Weyl group of same name for each RDP.

TheоRем. There exists a natural cartesian diagram

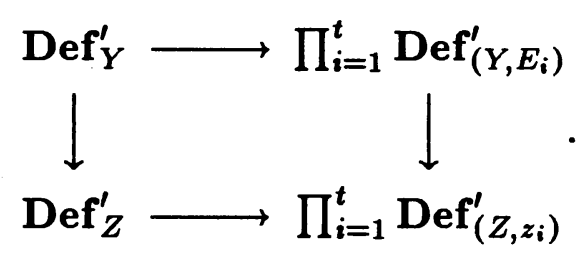

All spaces are smooth, the horizontal (restriction) maps are smooth, the vertical maps, induced by blowing down deformations, are surjective and Galois with Galois group the product of the Weyl groups $W_{i}$.

Proof: J. Wahl states and proves this theorem in the case where $Z$ is the rational double point resolution of a rational surface singularity, and $Y$ is the minimal resolution (cf. [Wahl 2,Theorem 1.3] and [Burns-Wahl, Theorems 2.6 and 2.14]). Suitably changed his argument applies here too.

By [Burns-Wahl,Theorem 2.6] we are guaranteed that the diagram is cartesian, once we have checked that the map $g$ is equivariant: $g_{*} \Theta_{Y}=\Theta_{Z}$. Equivariance is needed to lift local infinitesimal automorphisms from $Z$ to $Y$. 
Equivariance of $g$ follows from 2.3.3 by taking invariants of the map $g_{*} \Theta_{B} \rightarrow \Theta_{A}$ associated with the contraction $B \rightarrow A$. Note that this contraction factors the minimal resolution $M(2, \ldots, 2) \rightarrow A$ of the rational double point $A \cong A_{r s-1}$, so by equivariance of the minimal resolution it is equivariant too. Since tangent sheaves are reflexive $\left(\Theta_{B}\right)^{\mathbf{Z}_{r}} \cong$ $\Theta_{Y}$ and $\left(\Theta_{A}\right)^{\mathbf{z}_{r}} \cong \Theta_{X}$.

To assure smoothness of the horizontal maps we have to show surjectivity on the tangent space level. Looking at the bottom row we apply the local to global spectral sequence of cotangent cohomology.

$$
T_{Z}^{1} \rightarrow H^{0}\left(Z, \mathcal{T}_{Z}^{1}\right) \rightarrow H^{2}\left(\Theta_{Z}\right)=0 .
$$

Hence the natural forgetful map from the tangent space $T_{Z}^{1}$ of Def D $_{Z}$ to the tangent space $\oplus_{i=1}^{t} T_{\left(Z, z_{i}\right)}^{1}$ of $\prod_{i=1}^{t} \operatorname{Def}_{\left(Z, z_{i}\right)}$ is surjective. In particular by restriction we get the surjectivity of the bottom horizontal map.

For the top horizontal map let the singular points $z_{1}, \ldots, z_{t}$ of $Z$ be ordered in such a way that $z_{1}, \ldots, z_{k}$ are the rational double points, and $z_{k+1}, \ldots, z_{t}$ are of type $T$. Then for $i=1, \ldots, k$ the sheaf $\mathcal{T}_{Y}^{1} \mid Y_{i}=0$. By Lemma 2.4.1 the cohomology $H^{1}\left(Y_{i}, \Theta_{Y_{i}}\right)=0$, $i=k+1, \ldots, t$, and as an immediate consequence $H_{E_{i}}^{1}\left(Y_{i}, \Theta_{Y_{i}}\right)=0$ for $i=k+1, \ldots, t$.

There is the usual short exact sequence

$$
0 \rightarrow H^{1}\left(Y, \Theta_{Y}\right) \rightarrow T_{Y}^{1} \rightarrow H^{0}\left(Y, \mathcal{T}_{Y}^{1}\right) \rightarrow 0
$$

and the forgetful map obviously surjects the right hand side onto

$$
\oplus_{i=k+1}^{t} H^{0}\left(Y_{i}, \mathcal{T}_{Y_{i}}^{1}\right)=\oplus_{i=1}^{t} H^{0}\left(Y_{i}, \mathcal{T}_{Y_{i}}^{1}\right)
$$

Hence it suffices to show that $H^{1}\left(Y, \Theta_{Y}\right)$ maps onto $\oplus_{i=1}^{t} H^{1}\left(Y_{i}, \Theta_{Y_{i}}\right)$. For the type $T$ chains

$$
H^{1}\left(Y_{i}, \Theta_{Y_{i}^{\prime}}\right)=H_{E_{i}}^{1}\left(\Theta_{Y}\right)=0
$$

by Lemma 2.4.1, and we can conclude as in [Burns-Wahl,Theorem 2.14].

Finally the right hand side of the diagram is Galois as required. For the rational double points we refer to Brieskorn's construction [Brieskorn], and the type $T$ case we did in Section 2.

3.4. Let $Y \stackrel{g}{\rightarrow} Z \stackrel{f}{\rightarrow} X$ be as above and let $\mathcal{Y}^{\prime}$ be the family over $\operatorname{Def}_{Y}^{\prime}$. The general fibre $\mathcal{Y}_{t}^{\prime}$ is smooth, and maps isomorphically to the fibre over the image of $t$ in $S \subset \operatorname{Def}_{X}$. Since the singularities of $Y$ are of type $T_{0}$ the second homology $H_{2}\left(\mathcal{Y}_{t}^{\prime}, Q\right)$ is the same as $H_{2}(Y, Q)$. The latter is generated by the classes of the irreducible components of the exceptional set.

A divisor $C$ on $Y$ is called (as in \$2) a root if $C^{2}=-2$. It is called an irreducible root if $|C|$ is irreducible. By 3.2 the irreducible roots on $Y$ are either irreducible components of RDP configurations or the irreducible roots of $\S 2$.

The following theorem follows from the above remarks, 2.5.2 and 3.3.2. (Statement 3 for RDP configurations is handled in [Wahl 3].) 
THEOREM. Let $S$ be the smoothing component in the semiuniversal base space of a quotient surface singularity $X$, determined by a $P$-resolution $f: Z \rightarrow X$, and let $g: Y \rightarrow Z$ be the crepant $M$-resolution.

(1) The second homology $H_{2}\left(\mathcal{X}_{t}, Q\right)$ of the general fibre over $S$ is isomorphic to the homology $\mathrm{H}_{2}(Y, Q)$, and has a basis consisting of the irreducible exceptional curves on $Y$.

(2) The monodromy group is the product of the special monodromy groups of the singularities on the $P$-resolution $Z$, and it acts as a reflection group, generated by the roots on $Y$ (which are exactly the $g$-exceptional components).

(3) The reflection hyperplanes of $W$ are exactly the subspaces where a root lifts.

REMARK: Let $\mathcal{X}^{\prime}$ be the contraction of $\mathcal{Y}^{\prime}$ over $\operatorname{Def}_{Y}^{\prime}$. The discriminant of the component $S$ is the image of the discriminant of $\mathcal{X}^{\prime} \rightarrow$ Def $_{Y}^{\prime}$. One can show that the codimension one part of this discriminant is the discriminant of $\mathcal{Y}^{\prime} \rightarrow \operatorname{Def}_{Y}^{\prime}$ union the reflection hyperplanes. Notice that only the loops around the images of reflection hyperplanes act as non-trivial monodromy.

\section{§4. ROOTS AND FLOPS AND THE CYCLIC CASE.}

4.1. We give a geometric explanation for the identification of $W$-reflected points in Def . $_{\text {. }}$ As in the case of the Artin component, these reflections are related to the flops introduced in recent 3-fold theory.

4.1.1. Details on flops may be found in [Kollár], we recall the definition. A three dimensional curve neighborhood is a pair $C \subset X$ where $C$ is a proper connected curve and $X$ is the germ of a normal threefold along $C$. It is contractible if there is a morphism $f:(C \subset X) \rightarrow(P \in Y)$, where $Y$ is the germ of a normal singularity around the point $P$, such that $f(C)=P$ and $f: X \backslash C \rightarrow Y \backslash P$ is an isomorphism.

Given such a contractible curve neighborhood, assume $X$ has canonical singularities and that $K_{X} \mid C=0$. Let $H$ be a line bundle on $X$ such that $H^{-1} \mid C$ is ample. A three dimensional curve neighborhood $C^{+} \subset X^{+}$, bimeromorphic to $C \subset X$, is called the flop of $C \subset X$ (with respect to $H$ ) if there exists a line bundle $H^{+}$on $X^{+}$such that $H^{+} \mid C^{+}$ is ample.

It is proven, that under the conditions of the definition, flops do exist and they are unique. In the case of terminal singularities they are also independent of the choice of $H$.

4.1.2. Let us first look at the case of the $\mathrm{qG}$-component of a type $T$ singularity $X$. We use the notation of $\S 2$. Fix a root $C$ on the M-resolution $Y$ and the reflection hyperplane $R=R_{C} \subset \operatorname{Def}_{Y}^{0}$ belonging to the corresponding reflection $\sigma$. For each point $p \in R$ we have a line $L_{p}$ through $p$ and normal to $R$. By construction the the family of surfaces $V_{p}$ over $L_{p}$ is a normal threefold with only terminal singularities. The family of threefolds $\mathcal{V}=\bigcup V_{p} \rightarrow R$ is a flat deformation of $V_{0}$.

By definition $C$ lifts over $R$ to $\mathcal{C} \subset \mathcal{V}$, so we have a preferred curve $C_{p}$ in each $V_{p}$. A calculation shows that the contraction to a deformation of $X$ described in 2.4.2 is exactly contraction of $\mathcal{C}$. The involution on $L_{p}, \sigma(p)=p^{+}$yields the flop of $C_{p} \subset V_{p}$, namely $C_{p}+\subset V_{p+}$. Moreover, for these curve neighborhoods $C_{p} \subset V_{p}$ is isomorphic to the flop $C_{p}+\subset V_{p}+$ thereof the identification in $\operatorname{Def}_{X}$. 
The proof of these statements is a calculation using the description in $§ 2$ and comparison with the list of all possible flops in [Kollár,2.2.2.1].

4.1.3. In general the roots are either as described above or come from RDP configurations on the M-resolution (3.2). The latter case is treated in e.g. [Wahl 2, 1.4] in the case of the minimal resolution, but there is no problem extending the argument to M-resolution in general. Also it is easy to see the that the so called "elementary operation" described there is a flop. (See e.g. example 2.1.4.1 in [Kollár].) Since in the general case $W$ is a product of reflection groups determined by these two local pictures we get the relation to flops by decomposing.

4.2. We compute the monodromy for cyclic quotient singularities. The following is a corollary of Stevens' method for proving Theorem 1.3.2.

ThEOREM. For $[\mathbf{k}] \in K_{e-2}(X)$, there are at most $e-2$ singularities on the corresponding $P$-resolution $Z([\mathbf{k}]), Z_{1}, \ldots, Z_{e-2}$ and they satisfy

$$
Z_{i}=A_{\alpha_{i}\left(a_{i}-k_{i}\right)-1} / \mathbf{Z}_{\alpha_{i}}
$$

where our convention is

(1) $A_{r s-1} / Z_{r}$ with $r \geq 2, s \geq 1$ is a type $T$ singularity of index $r$ and canonical cover $A_{r s-1}$.

(2) $A_{s-1} / \mathbf{Z}_{1}$ with $s \geq 1$ is an $A_{s-1}$ singularity ( $A_{0}$ is a smooth point).

(3) $A_{-1} / \mathbf{Z}_{r}$ is a smooth point.

REMARK: The " $d$ " needed to completely determine the type $T$ singularity in (1) may also be computed from [a] and [k], but we will not need this.

Theorem 3.4 gives us the following

COROLlaRY. For $[\mathbf{k}] \in K_{e-2}(X)$, the monodromy group of the corresponding component is $\prod_{i=1}^{e-2} \mathbf{S}_{a_{i}-k_{i}}$ where $\mathbf{S}_{n}$ is the symmetric group on $n$ letters.

This was the covering group observed in [Christophersen 1] so we have proven the conjecture mentioned in the introduction.

\section{APPENDIX}

We treat the problem of lifting divisors to deformations of normal surfaces. Most of this can be found in a much more general setting in the literature on deformation theory, e.g. [Laudal]. We omit therefore proofs and do not explain the standard notation of the cotangent complex groups and sheaves. (We distinguish between the groups $T^{i}$ and sheaves $\mathcal{T}^{i}$ with notation.)

Consider a Weil divisor $Z$ on a normal surface $X$ and the sub-deformation functor Def $_{X, Z}$ of deformations of $X$ to which $Z$ lifts. That is deformations $f: \mathcal{X} \rightarrow S$ such that there exists $\mathcal{Z} \subset \mathcal{X}$ with $f: \mathcal{Z} \rightarrow S$ flat and $\mathcal{Z}_{0}=Z$.

There are natural maps $T_{X}^{i} \rightarrow T_{X}^{i}\left(\mathcal{O}_{Z}\right)$ and a long exact sequence

$$
\cdots \rightarrow T_{Z / X}^{i} \rightarrow T_{Z}^{i} \rightarrow T_{X}^{i}\left(\mathcal{O}_{Z}\right) \rightarrow T_{Z / X}^{i+1} \rightarrow \cdots
$$


In particular in low degree we have a diagram with exact rows

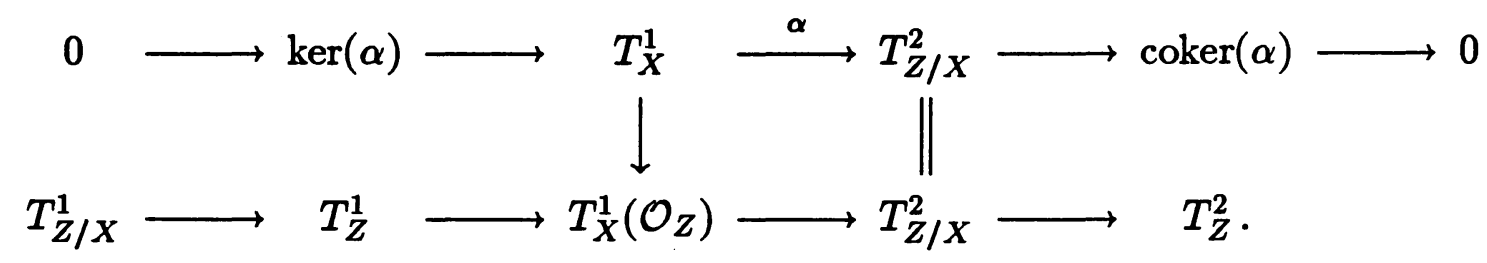

It is clear from the diagram that the tangent space of $\operatorname{Def}_{X, Z}$ is $\operatorname{ker}(\alpha)$ and it also true that the obstruction space is $\operatorname{coker}(\alpha)$.

In general for a scheme $Y$ over $S$ there is a spectral sequence

$$
H^{p}\left(Y, \mathcal{T}_{Y / S}^{q}(M)\right) \Rightarrow T_{Y / S}^{p+q}(M)
$$

where $M$ is an $\mathcal{O}_{Y}$ module. In our case this yields a commutative diagram with exact rows

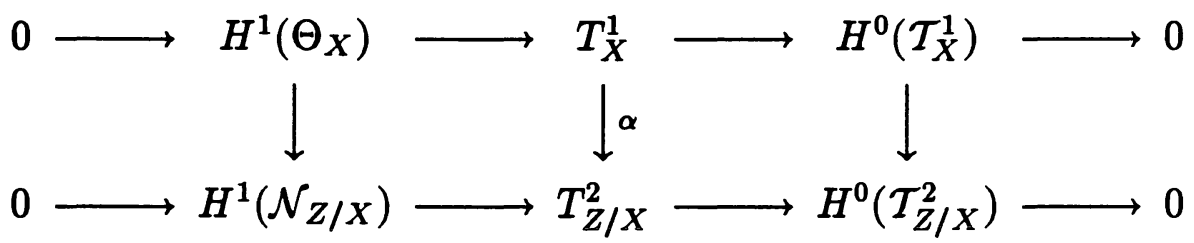

$\left(\mathcal{T}_{X}^{0}=\Theta_{X}\right.$ and $\mathcal{T}_{Z / X}^{1}=\mathcal{N}_{Z / X}$ the normal sheaf of $Z$ in $\left.X\right)$. If $X$ is smooth and $Z$ Cartier then the groups on the right are both trivial and we retrieve the well known description of Def $_{X, Z}$ in terms of the natural map $H^{1}\left(\Theta_{X}\right) \rightarrow H^{1}\left(\mathcal{N}_{Z / X}\right)$.

Proposition. Let $X$ be a normal surface and $Z$ a divisor on $X$. Let $U \subseteq$ Def $_{X}$ be a smooth subspace, $R_{Z}=\operatorname{Def}_{X, Z} \cap U$ and $T_{0} U \subseteq T_{X}^{1}$ the tangent space of $U$ at the special point. Assume

(1) $T_{0} U \subseteq \operatorname{ker}\left(T_{X}^{1} \rightarrow H^{0}\left(\mathcal{T}_{Z / X}^{2}\right)\right)$, i.e. there are no local obstructions for lifting $Z$.

(2) $h^{0}\left(\mathcal{N}_{Z / X}\right)=0(Z$ is rigid in $X)$ and $h^{1}\left(\mathcal{N}_{Z / X}\right)=1$.

(3) The restriction of $\alpha$ to $T_{0} U$ is not the zero map.

Then if $Z$ lifts it lifts uniquely and $R_{Z}$ is a hyperplane in $U$.

ProOF: The above spectral sequence yields an exact sequence

$$
0 \rightarrow H^{1}\left(\Theta_{Z / X}\right) \rightarrow T_{Z / X}^{1} \rightarrow H^{0}\left(\mathcal{N}_{Z / X}\right) \rightarrow 0
$$

so by assumption $T_{Z / X}^{1}=0$. Thus we get an induced map $\operatorname{ker}(\alpha) \rightarrow T_{Z}^{1}$ showing that the lifting is unique up to first order. But from the assumptions $\alpha$ induces a surjection $T_{0} U \rightarrow H^{1}\left(\mathcal{N}_{Z / X}\right)$ with codimension 1 kernel. Thus the liftings are unobstructed and happen over a hyperplane. 


\section{REFERENCES}

[AGV] Arnold, V.I.,Gusein-Zade, S.M.,Varchenko, A.N., "Singularities of differentiable mappings, II," Monographs in Mathematics, 83,, Birkhäuser, Boston, Basel, Berlin,, 1988.

[Arndt] Arndt, J., "Verselle Deformationen zyklischer Quotientensingularitäten," Dissertation, Universität Hamburg, 1988.

[Artin] Artin, M., Algebraic construction of Brieskorns resolutions, J. of Algebra 29 (1974), 330-348.

[Brieskorn] Brieskorn, E., Die Auflösung der Singularitäten holomorpher Abbildungen, Math. Ann. 178 (1968), 255-270.

[Briney] Briney, R.E., Intersection theory on quotients of algebraic varieties, Am. J. of Math. 84 (1962), 217-238.

[Burns-Wahl] Burns,D., Wahl, J., Local contributions to global deformations of surfaces, Inventiones Math. 26 (1974), 67-88.

[Christophersen 1] Christophersen, J.A., "Obstructions for rational singularities and deformations of cyclic quotients," Thesis, Universitetet i Oslo, 1990.

[Christophersen 2] On the components and discriminant of the versal base space of cyclic quotient singularities, in "Proc. Warwick Symposium on Singularity Theory and Applications, Vol. 1" (to appear).

[Christophersen 3] Deformations of Hypersurface Sections, (to appear).

[Giraud] Giraud, J., Intersections sur les surfaces normales, in "Introduction à la théorie des singularités II: Méthodes algébriques et géométriques," Hermann, Paris, 1988, pp. 69-96.

[Knöller] Knöller,F.W., 2-dimensionale Singularitäten und Differentialformen, Math. Ann. 206 (1973), 205-213.

[Kollár] Kollár, J., Flips, Flops, Minimal Models etc., Preprint (1989).

[Kollár-Shepherd-Barron] Kollár, J. , Shepherd-Barron, N. I., Threefolds and deformations of surface singularities, Inventiones Math. 91 (1988), 299-338.

[Laudal] Laudal, O.A., "Formal moduli of algebraic structures," Lecture Notes in Mathematics 754, Springer-Verlag, Berlin-Heidelberg-New York.

[Laufer] Laufer, H., "Normal two-dimensional singularities," Annals of Math. Studies 71, Princeton Univ. Press, 1971.

[Lipman] Lipman, J., Double point resolutions of deformations of rational singularities., Compositio Math. 88 pages 37-42 (1979.).

[Looijenga-Wahl] Looijenga, E., Wahl, J., Quadratic functions and smoothing surface singularities, Topology 25 (1986), 261-291.

[Riemenschneider] Riemenschneider, O., Deformationen von Quotientensingularitäten (nach zyklischen Gruppen), Math. Ann. 209 (1974), 211-248.

[Schlessinger] Schlessinger, M., Rigidity of quotient singularities, Inventiones Math. 14 (1971), 17-26.

[Stevens] J. Stevens, On the versal deformation of cyclic quotient singularities, in "Proc. Warwick Symposium on Singularity Theory and Applications, Vol. 1" (to appear).

[Tjurina] Tjurina, G.N., Resolutions of singularities of flat deformations of rational double points, Funct. Anal. Appl. 4 (1970), 77-83.

[Wahl 1] Wahl, J., Elliptic deformations of minimally elliptic singularities, Math. Ann. 253 (1980), 241-262.

[Wahl 2] Simultaneous resolution of rational singularities, Compositio Math. 38 (1979), 43-54.

[Wahl 3] 341-375. Simulaneous resolution and discriminantal loci, Duke Math. Journal 46 (1979),

Mathematisches Seminar Universität Hamburg, Bundesstrasse 55, D-2000 Hamburg 13 Matematisk Institutt, PB 1053 Blindern, 0316 Oslo 3 , Norway 